\title{
Clinical and Molecular Epidemiology of Extended-Spectrum $\beta$-lactamase- Producing Klebsiella pneumoniae and Escherichia Coli in a Japanese Tertiary Hospital
}

Yosuke Harada ${ }^{1,2}$, Yoshitomo Morinaga*1, Koichi Yamada ${ }^{1,2}$, Yohei Migiyama ${ }^{1,2}$, Kentaro Nagaoka ${ }^{1,2}$, Naoki Uno ${ }^{1}$, Shigeki Nakamura $^{2}$, Yoshifumi Imamura², Taiga Miyazaki², Hiroo Hasegawa' ${ }^{1}$, Koichi Izumikawa ${ }^{2}$, Hiroshi Kakeya ${ }^{2}$, Katsunori Yanagihara ${ }^{1}$ and Shigeru Kohno ${ }^{2,3}$

${ }^{1}$ Department of Laboratory Medicine, Japan

${ }^{2}$ Second Department of Internal Medicine, Nagasaki University Graduate School of Biomedical Sciences, Nagasaki, Japan

${ }^{3}$ Global COE Program, Nagasaki University, Nagasaki, Japan

\begin{abstract}
The increase in the incidence of extended-spectrum $\beta$-lactamase (ESBL)-producing bacteria has become a serious problem worldwide, but the distribution of ESBL-producing bacteria can vary according to geographical area or institution. The aim of this study was to analyze epidemiologic data on ESBL-producing bacteria and their genotypes in our hospital. The hospital microbiology laboratory databases were reviewed for ESBL-producing Escherichia coli and Klebsiella pneumoniae from 2006 to 2010. The ESBL-producers were also molecularly analyzed and included the CTX-M, TEM and SHV genes. In a 5-year study, there were 1359 isolates of E. coli and 725 isolates of $K$. pneumoniae. Incidence of ESBL-producing E. coli and K. pneumoniae increased from $5.5 \%$ and $0.5 \%$ in 2006 to $20.4 \%$ and $4.7 \%$ in 2010 . One half of the ESBL-producing E. coli was positive for at least $2 \mathrm{ESBL}$ genes. The most common genotype was TEM+CTX-M (48.8\%) for E. coli and TEM+SHV $(30.0 \%)$ for K. pneumoniae. The MIC $\mathrm{C}_{50}$ of ceftriaxone and cefepime in the CTX-M or TEM/SHV+CTX-M type ESBL-producing $E$. coli were higher than those in the TEM/SHV type isolates. The increase of bacteria with multiple ESBL genes may be an emergent problem. Therefore, ESBL genotyping is needed for monitoring the important ESBLs that can lead to treatment failure and contribute to the appropriate use of antimicrobial agents and infection control.
\end{abstract}

Keywords: Extended-spectrum $\beta$-lactamase; Escherichia coli; Klebsiella pneumonia; TEM; SHV; CTX-M

\section{Introduction}

The emergence of extended-spectrum $\beta$-lactamase (ESBL)producing bacteria, particularly Escherichia coli and Klebsiella pneumoniae, is now a critical concern for the development of therapies against bacterial infection. Since the early 1980s, the number of nosocomial infections by ESBL-producing, gram-negative bacteria has been increasing worldwide, and $\beta$-lactamase production has become a major causative factor for increasing resistance to antibiotics [1-3]. The ESBL genes are mostly plasmid encoded [4], and most ESBLs can be divided into 3 genotypes: TEM, SHV, and CTX-M [5]. The major ESBL producer was K. pneumoniae before 2000, and the predominant ESBL genotypes were TEM and SHV [1]. E. coli has now become an important ESBL carrier in Western countries. In addition, a genotype CTX-M has become more prevalent worldwide compared to the TEM and SHV genotypes [1]. During the 1990s, ESBL-producing organisms were described mainly as members of the TEM- and SHV- $\beta$-lactamase families in E. coli and K. pneumoniae causing nosocomial outbreaks [6]. In the 1990s, a novel type of ESBL, the CTX-M enzyme, emerged worldwide [6]. The CTX-M types, now exceeding 50 different types, can be divided into 5 groups based on their amino acid identities: CTX-M-1, CTX-M-2, CTX-M-8, CTX-M-9, and CTX-M-25 [7]. It is clear that various CTX-M type ESBLs have spread worldwide and that specific CTX-M subgroups have been localized to different geographic areas $[1,8,9]$. Thus, in Japan, it is known that the CTX-M-8 and CTX-M-25 groups of enzymes are rarely found [10]. In Japan, since the first isolate of ESBL-producing bacterium in 1993 [11], the detection rates of ESBL-producers have been much lower than those in the rest of Asia; however, the increase in the incidence of ESBL producers remains a common issue $[12,13]$. Because the current trend of both bacteria in Japan is unknown and few studies with longitudinal observations of the nosocomial spread of these bacteria have been reported [14], we investigated epidemiologic data on the ESBL-producing E. coli and $K$. pneumoniae in our hospital and the ESBL genotypes. In addition, the antimicrobial susceptibilities of the ESBL producers were examined.

\section{Materials and Methods}

\section{Study design}

The present study was conducted at Nagasaki University Hospital located in southwestern Japan, which has 861 beds. Hospital microbiology laboratory databases from January 2006 to December 2010 were reviewed, and clinical isolates of E. coli and K. pneumoniae from specimens, except for feces, were analyzed for bacteriological and molecular epidemiology. The bacteria were identified using the Vitek-2 system (bioMerieux Japan Ltd., Tokyo, Japan) or the BD Phoenix ${ }^{\mathrm{TM}}$ Automated Microbiology System (BD Diagnostic Systems, Sparks, $\mathrm{MD})$. For the isolates identified by Vitek-2, additional susceptibility testing was performed. When several strains with ESBL were detected from the same patient, only 1 sample was counted as an ESBL isolate.

*Corresponding author: Yoshitomo Morinaga, MD, PhD, Department of Laboratory Medicine, Nagasaki University Graduate School of Biomedical Sciences, 1-7-1 Sakamoto, Nagasaki 852-8501, Japan, Tel: +81-95-819-7574; Fax: +81-95-819-7422; E-mail: y-morina@nagasaki-u.ac.jp

Received June 28, 2013; Accepted September 03, 2013; Published September 06, 2013

Citation: Harada Y, Morinaga Y, Yamada K, Migiyama Y, Nagaoka K, et al (2013) Clinical and Molecular Epidemiology of Extended-Spectrum $\beta$-lactamaseProducing Klebsiella pneumoniae and Escherichia Coli in a Japanese Tertiary Hospital. J Med Microb Diagn 2: 127. doi:10.4172/2161-0703.1000127

Copyright: $\odot 2013$ Harada Y, et al. This is an open-access article distributed under the terms of the Creative Commons Attribution License, which permits unrestricted use, distribution, and reproduction in any medium, provided the original author and source are credited. 
Citation: Harada Y, Morinaga Y, Yamada K, Migiyama Y, Nagaoka K, et al. (2013) Clinical and Molecular Epidemiology of Extended-Spectrum $\beta$-lactamase-Producing Klebsiella pneumoniae and Escherichia Coli in a Japanese Tertiary Hospital. J Med Microb Diagn 2: 127. doi:10.4172/2161-0703.1000127

Information on the isolated strains, including etiology and susceptibility to antibiotics, was also obtained.

\section{Antimicrobial susceptibility testing}

Information on the susceptibility of the bacterial strains that had been performed according to the Clinical and Laboratory Standard Institute (CLSI) was obtained from the hospital microbiology laboratory databases [15]. The analyzed drugs were the following 11 agents: penicillin, ceftazidime, ceftriaxone, cefepime, imipenem, meropenem, aztreonam, gentamicin, minocycline, ciprofloxacin, and levofloxacin.

\section{Detection of ESBL-producing E. coli and K. pneumonia}

The isolates identified by $\mathrm{BD}$ Phoenix ${ }^{\mathrm{TM}}$ were analyzed with the integrated BDXpert ${ }^{\mathrm{TM}}$ System [16]. These processes were performed according to the original algorithm [17]. For the isolates identified by Vitek-2, the confirmatory testing for ESBL was performed by cephalosporin plus clavulanic acid (CA). Confirmation of ESBL isolates was based on 8-fold reduction with CA when combined with cefpodoxime, cefotaxime and ceftazidime. The proportional reduction in growth in the wells containing cephalosporin plus CA compared with those containing cephalosporin alone was considered indicative of ESBL production.

\section{Extraction of plasmids}

A few colonies were suspended in $700 \mu \mathrm{L}$ of Tris-EDTA buffer ( $\mathrm{pH}$ 8.0). The suspensions were boiled for $10 \mathrm{~min}$ and subsequently centrifuged for $5 \mathrm{~min}$ at $13000 \mathrm{rpm}$. The supernatant, containing DNA, was transferred to new tubes and stored at $4^{\circ} \mathrm{C}$ for subsequent PCR analysis.

\section{Genotyping of ESBL}

PCR was performed using 5 sets of previous published primers to amplify type-specific ESBL genes, including CTX-M-1, CTX-M-2, CTX-M-9, TEM and SHV [18]. For detecting TEM or SHV genes, initial denaturation at $95^{\circ} \mathrm{C}$ for $10 \mathrm{~min}$, denaturation at $95^{\circ} \mathrm{C}$ for $1 \mathrm{~min}$, primer annealing at $56^{\circ} \mathrm{C}$ for $1 \mathrm{~min}$, and extension at $72^{\circ} \mathrm{C}$ for $1 \mathrm{~min}$, was repeated for 40 cycles; with a final extension at $72^{\circ} \mathrm{C}$ for $7 \mathrm{~min}$. For CTX-M gene, initial denaturation at $95^{\circ} \mathrm{C}$ for $10 \mathrm{~min}$, denaturation at $95^{\circ} \mathrm{C}$ for $1 \mathrm{~min}$, primer annealing at $60^{\circ} \mathrm{C}$ for $1 \mathrm{~min}$, and extension at $72^{\circ} \mathrm{C}$ for $1 \mathrm{~min}$, was repeated for 40 cycles; a final extension at $72^{\circ} \mathrm{C}$ for 7 min was carried out. The PCR products were analyzed using $2 \%$ agarose gel electrophoresis and visualized by staining with ethidium bromide.

\section{Results}

\section{Identification of ESBL-producing $E$. coli strains}

ESBL-producing E. coli accounted for 160 of 1359 isolates (11.8\%) and ESBL-producing K. pneumoniae accounted for 20 of 725 isolates (2.8\%). The number of ESBL-producing E. coli markedly increased from 17 isolates (6.8\%) in 2006 to 68 isolates (22.7\%) in 2010 (Figure 1). ESBL-producing K. pneumoniae was not detected in 2006, but appeared after 2007 and peaked in 2009 with 9 isolates (6.4\%). The majority of ESBL-producing E. coli were isolated from urine (59\%), followed by respiratory specimens (14\%), pus (12\%), blood (9\%) and others $(6 \%)$ (Figure 2).

\section{Molecular characterization of ESBL-producing strains}

The phenotypically identified ESBL-producing E. coli and $K$. pneumoniae were molecularly analyzed. Of the 160 ESBL-producing $E$. coli isolates, $143(89.4 \%)$ were positive for ESBL genes (Table 1). Fifty

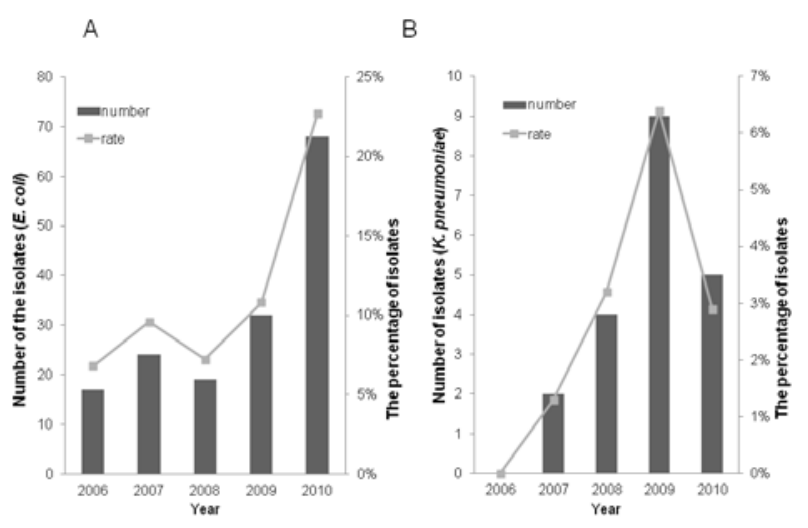

Figure 1: Frequencies of ESBL-producing organisms.

The number and the rate of ESBL-producing $E$. coli $(\mathrm{A})$ and $K$. pneumoniae (B) in our hospital

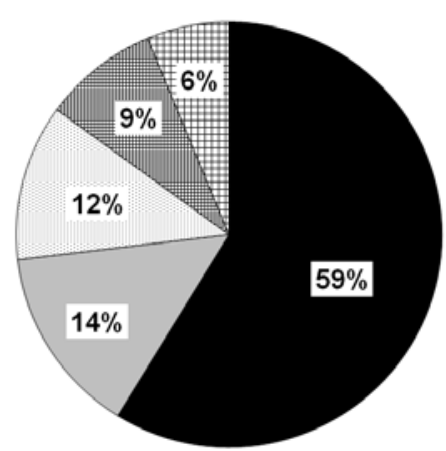

-urine $\square$ respiratory specimens $\square$ pus 由blood $\boxminus$ others

Figure 2: The origins of ESBL-producing $E$. coli.

The isolates were obtained from urine $(n=94,59 \%)$, respiratory specimens $(n=23,14 \%)$, pus $(n=19,12 \%)$, blood $(n=14,9 \%)$, and others $(n=10,6 \%)$

percent of the isolates had at least 2 ESBL genes. The number of ESBLproducing E. coli with TEM, SHV, CTX-M-1, CTX-M-2 and CTX-M-9 was $13(8.1 \%), 5(3.1 \%), 7(4.4 \%), 3(1.9 \%)$ and $35(21.9 \%)$, respectively (Table 1). The number of ESBL-producing E. coli with TEM+SHV, TEM+CTX-M-1, TEM+CTX-M-2, TEM+CTX-M-9, TEM+CTX-M1+CTX-M-9, TEM+CTX-M-1+CTX-M-2 and SHV+CTX-M-9 was 2 (1.3\%), 10 (6.3\%), 3 (1.9\%), 61 (38.1\%), 2 (1.3\%), 1 (0.6\%) and $1(0.6 \%)$, respectively. In addition, the detection number of ESBL-producing $E$. coli of the CTX-M-1 group increased from 1 to 11 from 2006 to 2010. For K. pneumoniae, 17 (85.0\%) of the 20 isolates were positive for ESBL genes (Table 2). The number of ESBL-producing $K$. pneumoniae with TEM, SHV, TEM+SHV, SHV+CTX-M-1, SHV+CTX-M-2, SHV+CTX-M-9 and TEM+SHV+CTX-M-1 was 1 (5\%), 5 (25\%), $6(30 \%), 1(5 \%), 1(5 \%), 2(10 \%)$, and $1(5 \%)$, respectively. ESBLproducing $K$. pneumoniae isolates carrying CTX-M alone were not detected.

\section{Antimicrobial susceptibilities of ESBL-producing $E$. coli}

We analyzed the antimicrobial susceptibilities of the ESBLproducing E. coli between genotypes (Table 3). The isolates were categorized according to the following genotypes: TEM/SHV (TEM and/or SHV), CTX-M (CTX-M-1, CTX-M-2, and CTX-M-9), and 
Citation: Harada Y, Morinaga Y, Yamada K, Migiyama Y, Nagaoka K, et al. (2013) Clinical and Molecular Epidemiology of Extended-Spectrum $\beta$-lactamase-Producing Klebsiella pneumoniae and Escherichia Coli in a Japanese Tertiary Hospital. J Med Microb Diagn 2: 127. doi:10.4172/2161-0703.1000127

\begin{tabular}{|l|c|c|c|c|c|c|}
\hline & \multicolumn{7}{|c|}{ Year } \\
\hline Genotype(s) & $\mathbf{2 0 0 6}$ & $\mathbf{2 0 0 7}$ & $\mathbf{2 0 0 8}$ & $\mathbf{2 0 0 9}$ & $\mathbf{2 0 1 0}$ & Total n (\%) \\
\hline TEM & 0 & 1 & 0 & 4 & 8 & $13(8.1)$ \\
\hline SHV & 2 & 0 & 0 & 1 & 2 & $5(3.1)$ \\
\hline TEM+SHV & 0 & 0 & 0 & 0 & 2 & $2(1.3)$ \\
\hline CTX-M-1 & 0 & 0 & 1 & 1 & 5 & $7(4.4)$ \\
\hline CTX-M-1+TEM & 1 & 1 & 1 & 1 & 6 & $10(6.3)$ \\
\hline CTX-M-2 & 0 & 0 & 1 & 0 & 2 & $3(1.9)$ \\
\hline CTX-M-2+TEM & 0 & 2 & 0 & 0 & 1 & $3(1.9)$ \\
\hline CTX-M-9 & 6 & 8 & 7 & 6 & 8 & $35(21.9)$ \\
\hline CTX-M-9+TEM & 7 & 10 & 9 & 11 & 24 & $61(38.1)$ \\
\hline CTX-M-1+CTX-M-2+TEM & 0 & 1 & 0 & 0 & 0 & $1(0.6)$ \\
\hline CTX-M-1+CTX-M-9+TEM & 0 & 0 & 0 & 2 & 0 & $2(1.3)$ \\
\hline CTX-M-9+SHV & 1 & 0 & 0 & 0 & 0 & $1(0.6)$ \\
\hline Undetected & 0 & 1 & 0 & 6 & 10 & $17(10.6)$ \\
\hline Total & 17 & 24 & 19 & 32 & 68 & 160 \\
\hline
\end{tabular}

Table 1: Genotypes of ESBL-producing E. coli from 2006 to 2010

\begin{tabular}{|l|c|c|c|c|c|c|}
\hline & \multicolumn{7}{|c|}{ Year } \\
\hline Genotype(s) & $\mathbf{2 0 0 6}$ & $\mathbf{2 0 0 7}$ & $\mathbf{2 0 0 8}$ & $\mathbf{2 0 0 9}$ & $\mathbf{2 0 1 0}$ & Total n (\%) \\
\hline TEM & 0 & 0 & 0 & 1 & 0 & $1(5)$ \\
\hline SHV & 0 & 1 & 1 & 2 & 1 & $5(25)$ \\
\hline TEM+SHV & 0 & 1 & 2 & 2 & 1 & $6(30)$ \\
\hline CTX-M-1+SHV & 0 & 0 & 0 & 1 & 0 & $1(5)$ \\
\hline CTX-M-2+SHV & 0 & 0 & 0 & 0 & 1 & $1(5)$ \\
\hline CTX-M-9+SHV & 0 & 0 & 1 & 1 & 0 & $2(10)$ \\
\hline CTX-M-1+TEM+SHV & 0 & 0 & 0 & 1 & 0 & $1(5)$ \\
\hline Undetected & 0 & 0 & 0 & 1 & 2 & $3(15)$ \\
\hline Total & 0 & 2 & 4 & 9 & 5 & 20 \\
\hline
\end{tabular}

Table 2: Genotypes of ESBL-producing K. pneumoniae from 2006 to 2010 .

\begin{tabular}{|l|c|c|c|c|c|c|}
\hline Antibiotic & \multicolumn{2}{|c|}{ TEM/SHV } & \multicolumn{2}{c|}{ CTX-M } & \multicolumn{2}{c|}{ TEM/SHV+CTX-M } \\
\hline & $\mathrm{MIC}_{50}$ & $\mathrm{MIC}_{90}$ & $\mathrm{MIC}_{50}$ & $\mathrm{MIC}_{90}$ & $\mathrm{MIC}_{50}$ & $\mathrm{MIC}_{90}$ \\
\hline Penicillin & $\geq 32$ & $\geq 32$ & $\geq 32$ & $\geq 32$ & $\geq 32$ & $\geq 32$ \\
\hline Ceftazidime & 2 & $\geq 32$ & 2 & $\geq 32$ & 2 & 16 \\
\hline Ceftriaxone & 2 & $\geq 32$ & $\geq 32$ & $\geq 32$ & $\geq 32$ & $\geq 32$ \\
\hline Cefepime & 1 & $\geq 32$ & 8 & $\geq 32$ & 8 & $\geq 32$ \\
\hline Imipenem & $\leq 0.5$ & $\leq 0.5$ & $\leq 0.5$ & $\leq 0.5$ & $\leq 0.5$ & $\leq 0.5$ \\
\hline Meropenem & $\leq 0.5$ & $\leq 0.5$ & $\leq 0.5$ & $\leq 0.5$ & $\leq 0.5$ & $\leq 0.5$ \\
\hline Aztreonam & 2 & $\geq 32$ & 4 & $\geq 32$ & 8 & $\geq 32$ \\
\hline Gentamicin & 2 & 16 & 1 & 16 & 4 & 16 \\
\hline Minocycline & 2 & 8 & 2 & 16 & 2 & 16 \\
\hline Ciprofloxacin & 8 & 8 & 8 & 8 & 8 & 8 \\
\hline Levofloxacin & 8 & 8 & 8 & 8 & 8 & 8 \\
\hline
\end{tabular}

Table 3: Antimicrobial susceptibilities of ESBL-producing E. coli.

TEM/SHV+CTX-M (TEM/SHV and CTX-M), as previously reported [8]. The value of $\mathrm{MIC}_{50}$ of TEM/SHV in ceftriaxone and cefepime were $2 \mu \mathrm{g} / \mathrm{mL}$ and $1 \mu \mathrm{g} / \mathrm{mL}$, respectively. The values of $\mathrm{MIC}_{50}$ of CTX-M or TEM/SHV+CTX-M in ceftriaxone and cefepime were $\geq 32 \mu \mathrm{g} /$ $\mathrm{mL}$ and $8 \mu \mathrm{g} / \mathrm{mL}$ respectively. All of the TEM/SHV- and CTX-Mproducing isolates retained favorable susceptibility to carbapenems. In all genotypes, the value of $\mathrm{MIC}_{50}$ in fluoroquinolones was $8 \mu \mathrm{g} / \mathrm{mL}$.

\section{Discussion}

In this study, we examined the current trend of ESBL-producing $E$. coli and K. pneumoniae in our hospital, as well as the ESBL genotypes and the antimicrobial susceptibilities. Our data, based on the clinical isolates collected over 5 years, suggested that the incidence of ESBLproducing E. coli and K. pneumoniae have increased. The prevalence of ESBL-producing bacteria has been on the rise, particularly in Asia compared to other regions. A study conducted in 2007 reported that the frequencies of ESBL-producing K. pneumoniae and E. coli isolates exceeded $30 \%$ in both bacterial populations [11]. The proportion of ESBL-producing isolates in Japan was $4.3 \%$ in E. coli and 3.1\% in $K$. pneumoniae in 2006 [13]. Thus, the geographical distribution can vary according to countries and institutions, although the prevalence of ESBL-producing bacteria is a global problem [19]. The prevalence of ESBL-producers in our study was lower than that reported by global surveillances [20] and in other Asian countries [21,22] but it was found to increase, implying that ESBL producers could become common drug-resistant bacteria in the near future. In addition, our study suggested that urine can be an important source of ESBL-producing $E$. coli as previously reported [23]. Worldwide, the predominant genotype of ESBL-producing E. coli has changed from TEM and/or SHV (TEM/ SHV) to CTX-M $[1,24]$, and the detection rate of CTX-M has increased dramatically [25]. In this study, CTX-M was found most frequently in ESBL-producing E. coli isolates, in particular, the CTX-M-9 group whose prevalence increased during the observation period. Our results were similar to previously reported Japanese trends on the increase of CTX-M type ESBL $[10,26]$, but different in that the increase of prevalence of CTX-M-9 was in combination with an increase in the prevalence of the TEM genotype. An ESBL-producing E. coli clone O25:H4-ST131 that often carries CTX-M-1-type ESBL has been spreading worldwide. Because of its resistance to fluoroquinolones and aminoglycosides, in addition to $\beta$-lactams, the increase of $E$. coli clone O25:H4-ST131 has become a serious problem [23]. We did not investigate any specific clones including $E$. coli clone O25:H4-ST131 in this study; however, the mild increase of CTX-M-1-type ESBL may imply the spreading of $\mathrm{O} 25: \mathrm{H} 4-\mathrm{ST} 131$. In fact, this clone has previously been found in Japan [26]; therefore, further examination will be required. In the present study, about one-half of the ESBL-producing $E$. coli isolates were molecularly confirmed to have 2 or more ESBL genes. The incidence of TEM+CTX-M-9 has increased remarkably in these last 2 years. These findings suggest that $E$. coli carrying multiple ESBL genes may be increasing. In contrast, those isolates from which these specific genes were not detected likely produce other types of enzymes that have yet to be investigated. Alternatively, some identification errors may be involved in our results because a high false-positivity rate of Phoenix identification system had been reported [27]. The most common ESBL genotype among the K. pneumoniae isolates was TEM+SHV, as opposed to a previous report from Japan [8] which showed that SHV+CTX-M was the most common. Because of the limited number of isolates in our hospital, it is difficult to place TEM+SHV as the predominant genotype of ESBL-producing K. pneumoniae. The potential for hydrolysis of $\beta$-lactams can vary according to the type of ESBL enzymes. CTX-M $\beta$-lactamase can elevate the MIC values of ceftriaxone, cefepime, and ceftazidime $[28,29]$. In the present study, the $\mathrm{MIC}_{50}$ of ceftriaxone and cefepime in E. coli with CTX-M or TEM/SHV+CTX-M was higher than in those E. coli with TEM/SHV. Among the E. coli with CTX-M, the $\mathrm{MIC}_{90}$ and $\mathrm{MIC}_{50}$ of ceftazidime in E. coli with TEM+CTX-M-9, the predominant ESBL in this study, were lower than other E. coli with TEM/ SHV+CTX-M (data not shown). CTX-M ESBLs can be also resistant to fluoroquinolones [30], but there were no significant differences in the MICs of fluoroquinolones between ESBL genotypes. Thus, ESBLs showed different hydrolysis potentials and some plasmids can possess other drug-resistant genes in addition to a $\beta$-lactamase gene. Therefore, ESBL genotyping can help in monitoring the important ESBLs that can lead to treatment failure and contribute to the appropriate use of antimicrobial agents and the infection control. In conclusion, ESBLproducing E. coli and K. pneumoniae were less frequent compared to 
Citation: Harada Y, Morinaga Y, Yamada K, Migiyama Y, Nagaoka K, et al. (2013) Clinical and Molecular Epidemiology of Extended-Spectrum $\beta$-lactamase-Producing Klebsiella pneumoniae and Escherichia Coli in a Japanese Tertiary Hospital. J Med Microb Diagn 2: 127. doi:10.4172/2161-0703.1000127

the global trend but were found to be increasing in a tertiary hospital. Two or more ESBLs were detected in many isolates by molecular analysis, and MIC of some cephalosporin's was elevated in the isolates with CTX-M-type ESBL. Considering regional variations and some easy-expanding clones, constant and careful surveillance is needed.

\section{References}

1. Cantón R, Coque TM (2006) The CTX-M beta-lactamase pandemic. Curr Opin Microbiol 9: 466-475

2. Pitout JD, Laupland KB (2008) Extended-spectrum beta-lactamase-producing Enterobacteriaceae: an emerging public-health concern. Lancet Infect Dis 8: 159-166.

3. Ramphal R, Ambrose PG (2006) Extended-spectrum beta-lactamases and clinical outcomes: current data. Clin Infect Dis 42 (Suppl 4): S164-172.

4. Nakamura T, Komatsu M, Yamasaki K, Fukuda S, Miyamoto Y, et al. (2012) Epidemiology of Escherichia coli, Klebsiella Species, and Proteus mirabilis strains producing extended-spectrum beta-lactamases from clinical samples in the Kinki region of Japan. Am J Cli Pathol 137(4): 620-626.

5. Paterson DL, Bonomo RA (2005) Extended-spectrum beta-lactamases: a clinical update. Clin Microbiol Rev 18: 657-686.

6. Bradford PA (2001) Extended-spectrum beta-lactamases in the 21st century: characterization, epidemiology, and detection of this important resistance threat. Clin Microbiol Rev 14: 933-951.

7. Bonnet R (2004) Growing group of extended-spectrum beta-lactamases: the CTX-M enzymes. Antimicrob Agents Chemother 48: 1-14.

8. Chong $\mathrm{Y}$, Yakusiji $\mathrm{H}$, Ito $\mathrm{Y}$, Kamimura $\mathrm{T}$ (2011) Clinical and molecula epidemiology of extended-spectrum beta-lactamase-producing Escherichia coli and Klebsiella pneumoniae in a long-term study Japan. Eur J Clin Microbio Infection Dis 30: 83-87.

9. Suzuki S, Shibata N, Yamane K, Wachino J, Ito K, et al. (2009) Change in the prevalence of extended-spectrum-beta-lactamase-producing Escherichia coli in Japan by clonal spread. J Antimicrob Chemother 63: 72-79.

10. Shibata N, Kurokawa H, Doi Y, Yagi T, Yamane K, et al. (2006) PCR classification of CTX-M-type beta-lactamase genes identified in clinically isolated gramnegative bacilli in Japan. Antimicrob Agents Chemother 50: 791-795.

11. Ishii $\mathrm{Y}$, Ohno A, Taguchi H, Imajo S, Ishiguro M, et al. (1995) Cloning and sequence of the gene encoding a cefotaxime-hydrolyzing class $\mathrm{A}$ betalactamase isolated from Escherichia coli. Antimicrob Agents Chemother 39: 2269-2275.

12. Yamaguchi K, Murakami M, Takahashi A, Ishii Y, Iwata M, et al. (2005) Nationwide surveillance of parenteral antibiotics containing meropenem activities against clinically isolated strains in 2004. Jpn J Antibiot 58: 655-689.

13. Yamaguchi K, Ishii Y, Iwata M, Yoshida H, Satoh T, et al. (2007) Nationwide surveillance of parenteral antibiotics containing meropenem activities agains clinically isolated strains in 2006. Jpn J Antibiot 60: 344-377.

14. Chong Y, Yakushiji H, Ito Y, Kamimura T (2010) Cefepime-resistant Gramnegative bacteremia in febrile neutropenic patients with hematological malignancies. Int J Infect Dis 14 Suppl 3: e171-e175.

15. Wayne PA (2004) National Committee for Clinical Laboratory Standards (NCCLS) Performance Standards for Antimicrobial Susceptibility Testing. 14 Informational Supplement. NCCLS.

16. Turng B, Votta M, Turner D, Pollitt J, Callihan D, et al. (2002) Detection of Extended Spectrum beta-Lactamase Among Enterobacteriaceae using Phoenix ${ }^{\mathrm{TM}}$ Automated Microbiology System with BDXpert ${ }^{\mathrm{TM}}$ System. ICAAC, San Diego, California, 2002
17. Sanguinetti M, Posteraro B, Spanu T, Ciccaglione D, Romano L, et al. (2003) Characterization of clinical isolates of Enterobacteriaceae from Italy by the BD Phoenix extended-spectrum beta-lactamase detection method. J Clin Microbio 41: $1463-1468$

18. Muratani T, Kobayashi T, Matsumoto T (2006) Emergence and prevalence of beta-lactamase-producing Klebsiella pneumoniae resistant to cephems in Japan. Int J Antimicrob Agents 27: 491-499.

19. Coque TM, Baquero F, Canton R (2008) Increasing prevalence of ESBLproducing Enterobacteriaceae in Europe. Euro Surveill 13.

20. Hawser SP, Bouchillon SK, Hoban DJ, Badal RE (2009) In vitro susceptibilities of aerobic and facultative anaerobic Gram-negative bacilli from patients with intra-abdominal infections worldwide from 2005-2007: results from the SMART study. Int J Antimicrob Agents 34: 585-588.

21. Lewis MT, Yamaguchi K, Biedenbach DJ, Jones RN (1999) In vitro evaluation of cefepime and other broad-spectrum beta-lactams in 22 medical centers in Japan: a phase trial comparing two annual organism samples. The Japan Antimicrobial Resistance Study Group. Diagn Microbiol Infect Dis 35: 307-315.

22. Yamaguchi K, Mathai D, Biedenbach DJ, Lewis MT, Gales AC, et al. (1990) Evaluation of the in vitro activity of six broad-spectrum beta-lactam antimicrobia agents tested against over 2,000 clinical isolates from 22 medical centers in Japan. Japan Antimicrobial Resistance Study Group. Diagn Microbiol Infect Dis 34: 123-134.

23. Shigemura K, Tanaka K, Adachi M, Yamashita M, Arakawa S, et al. (2011) Chronological change of antibiotic use and antibiotic resistance in Escherichia coli causing urinary tract infections. J Infect Chemother 17: 646-651.

24. Romero L, López L, Rodríguez-Baño J, Hernández JR, Martínez-Martínez L, et al. (2005) Long-term study of the frequency of Escherichia coli and Klebsiella pneumoniae isolates producing extended-spectrum beta-lactamases. Clin Microbiol Infect 11: 625-631.

25. Rossolini GM, D'Andrea MM, Mugnaioli C (2008) The spread of CTX-M-type extended-spectrum beta-lactamases. Clin Microbiol Infect 14 Suppl 1: 33-41.

26. Kuroda H, Yano H, Hirakata Y, Arai K, Endo S, et al. (2012) Molecula characteristics of extended-spectrum $\beta$-lactamase-producing Escherichia coli in Japan: emergence of CTX-M-15-producing E. coli ST131. Diagn Microbio Infect Dis 74: 201-203.

27. Fisher MA, Stamper PD, Hujer KM, Love Z, Croft A, et al. (2009) Performance of the Phoenix bacterial identification system compared with disc diffusion methods for identifying extended-spectrum beta-lactamase, AmpC and KPC producers. J Med Microbiol 58: 774-778.

28. Yu WL, Pfaller MA, Winokur PL, Jones RN (2002) Cefepime MIC as a predicto of the extended-spectrum beta-lactamase type in Klebsiella pneumoniae, Taiwan. Emerg Infect Dis 8: 522-524.

29. Paterson DL (2000) Recommendation for treatment of severe infections caused by Enterobacteriaceae producing extended-spectrum beta-lactamases (ESBLs). Clin Microbiol Infect 6: 460-463.

30. Pitout JD, Nordmann P, Laupland KB, Poirel L (2005) Emergence of Enterobacteriaceae producing extended-spectrum beta-lactamases (ESBLs) in the community. J Antimicrob Chemother 56: 52-59. 